\title{
The effect of smoking on cognition as measured by Cambridge Neuropsychological Test Automated Battery (CATNAB) and brain-derived neurotrophic factor plasma levels
}

Arwa Ali S. Al-Mshari, MBBS, MSc, Mona H. AlSheikh, MBBS, PhD, Rabia Latif, MBBS, PhD, Sadaf Mumtaz, MBBS, PhD.

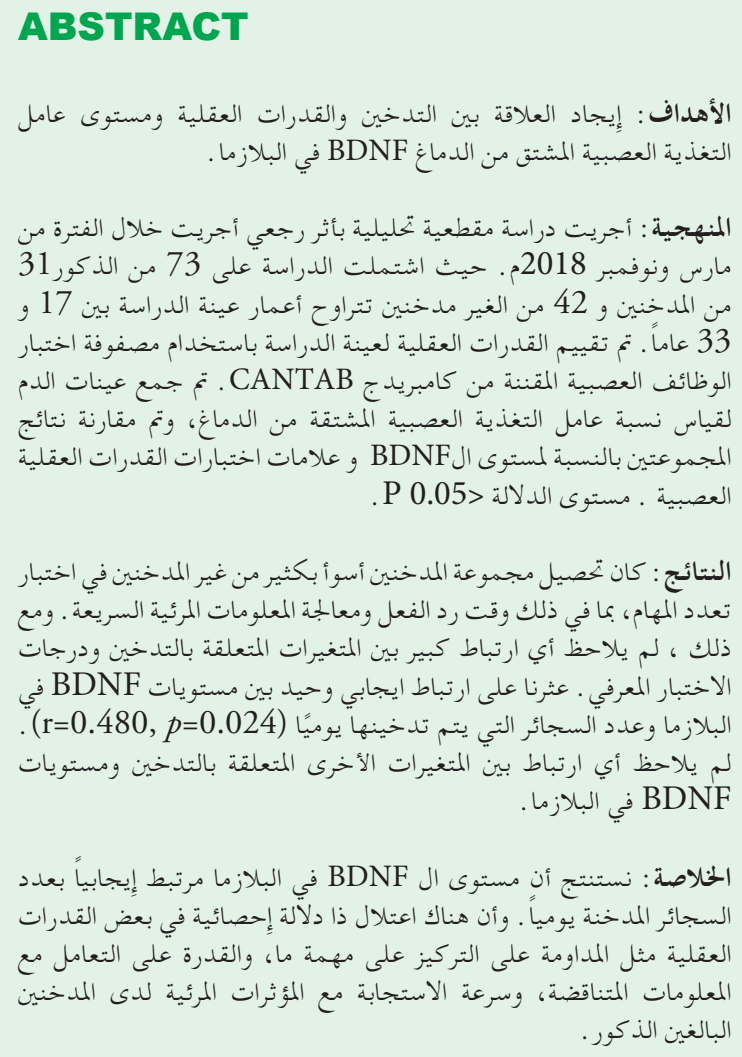

Objectives: To establish an association between cigarette smoking, cognition, and plasma brainderived neurotrophic factor (BDNF) levels in healthy young adults.

Methods: This was an ex post facto analytic crosssectional study conducted between March and November 2018. Participants were 73 healthy males (31 smokers and 42 non-smokers), 17-33 years old. The cognitive function of the participants was assessed through the Cambridge neuropsychological test automated battery (CANTAB). Blood samples were taken to measure the plasma levels of BDNF and the results were compared to identify the association between smoking related variables and cognitive test scores and plasma BDNF levels. A $p$-value of $<0.05$ was considered statistically significant.

Results: Smokers performed significantly worse than non-smokers in the multitasking test, including reaction time and rapid visual information processing. However, no significant association was observed between smoking related variables and cognitive test scores. The only significant positive correlation was found between plasma BDNF levels and the number of cigarettes smoked per day $(\mathrm{r}=0.480, p=0.024)$. No correlation was observed between other smoking related variables and plasma BDNF levels.

Conclusion: Plasma BDNF level is positively related to the number of cigarettes smoked per day. Young smokers have significantly impaired sustained attention and less ability to manage conflicting information as compared to age-matched nonsmokers.

Keywords: smoking, brain-derived neurotropic factor, cognition, neuropsychological tests, CATNAB

Saudi Med J 2020; Vol. 41 (12): 1308-1314 doi: 10.15537/smj.2020.12.25513

From the Department of Biomedical Sciences (Al-Mshari), College of Medicine, King Faisal University, Al-Ahsa; from the Department of Physiology (AlSheikh, Latif), College of Medicine, Imam Abdulrahman Bin Faisal University, Dammam, Kingdom of Saudi Arabia; and from the Department of Physiology (Mumtaz), Dental College, HITECInstitute of Medical Sciences, Taxilla, Pakistan.

Received 24th July 2020. Accepted 20th October 2020.

Address correspondence and reprint request to: Dr. Mona H. AlSheikh, Department of Physiology, College of Medicine, Imam Abdulrahman Bin Faisal University, Dammam, Kingdom of Saudi Arabia. E-mail:msheikh@iau.edu.sa

ORCID ID: https://orcid.org/0000-0003-3095-1969 
$\mathrm{T}$ There is currently a global prevalence of tobacco smoking of $21.2 \%$ among the adult population (35.8\% of males and $6.6 \%$ of females). ${ }^{1}$ The most recent World Health Organization's (WHO) global report on tobacco smoking found an increase in the prevalence of tobacco smoking is among both genders. Furthermore, the prevalence of tobacco smoking in the Kingdom Saudi Arabia (KSA) is projected to increase to $24 \%$ by $2025 .^{2}$

Tobacco smoking is known for its adverse impact on human health, resulting in the deaths of approximately 7 million individuals each year, with over 6 million of these arising from direct tobacco use and approximately 890,000 from passive smoking. ${ }^{3}$ A considerable degree of research has highlighted the deleterious effects of tobacco smoking, along with its association with multiple types of cancers, strokes, pulmonary and cardiovascular diseases, and reduced levels of immunity. ${ }^{4}$ The high concentration of free radicals in tobacco smoke can increase oxidative stress and damage to deoxyribonucleic acid and other neuronal structures. Furthermore, exposure to tobacco smoke increases oxidative stress by decreasing antioxidants important for maintaining oxidative balance. ${ }^{5}$ Smoking can also reduce cerebral perfusion, either directly (by affecting the vasomotor reactivity of cerebral arteries) or indirectly (by accelerating atherosclerosis formation). ${ }^{6}$ Smoking also increases homocysteine level in plasma, which is a known risk factor for cognitive impairment and stroke. ${ }^{7}$ In addition, tobacco smoking may cause cortical thinning and decrease grey matter density in brain areas significant for cognition, such as, the frontal lobe, thalamus and cerebellum. ${ }^{8}$ Furthermore, nicotine (an important chemical agent in tobacco), is known for its influence on cognition through stimulating nicotinic acetylcholine receptors distributed throughout the brain. ${ }^{9}$ Smoking-induced cognitive impairment has been reported even in passive smokers. ${ }^{10}$

The brain-derived neurotrophic factor (BDNF) has been proposed to have a significant role in brain development, synaptic plasticity, cognition and memory. ${ }^{11}$ A study showed higher BDNF levels in aged mouse as compared to young and BDNF levels were inversely correlated with the spatial memory. ${ }^{12}$ The significance of BDNF in the normal development and functioning of nervous system highlights the

Disclosure. Authors have no conflict of interests, and the work was not supported or funded by any drug company. importance of examining its association with tobacco smoking. Although, an association between chronic smoking and cognitive impairment in elderly and middle-aged subjects is well established, ${ }^{13}$ the impact of smoking on cognition of young individuals has remained controversial. This current research therefore focuses on exploring the association between smoking, cognition, and BDNF.

Methods. This study took the form of an ex post facto analytic cross-sectional study, conducted between March and November 2018 at the Department of Physiology, in College of Medicine, Imam Abdulrahman Bin Faisal University, Dammam, KSA.

The calculated sample size was 110 participants (55 smokers and 55 non-smokers). The calculation of the sample size employed OpenEpi, an open source epidemiologic statistics for public health (version 3.01) online tool. ${ }^{14}$ The calculation of the sample size was based on the mean scores of spatial working memory strategy (SWMS) and standard deviation (SD) values with a power of $80 \%$ and a $95 \%$ confidence interval.

We recruited 73 participants $(31$ smokers and 42 non-smokers) through purposive sampling (nonprobability sampling). Participants were recruited from Family and Community Medicine Center, Imam Abdulrahman bin Faisal University (IAU) and an antismoking clinic in Al Khobar, KSA.

Inclusion criteria were healthy males aged between 17 and 33 years old. We chose to employ only male participants due to it being simpler to recruit males than females, particularly as the prevalence of male smokers in KSA is far higher than females. ${ }^{2}$ The selected participants had a history of smoking at least one cigarette a day over the previous 6 months or more, regardless of the number/duration of previous attempts to give up smoking. The non-smoking participants were those who had never smoked tobacco in their lifetime.

We excluded subjects who had previously smoked but had given up at the time of the study. It also excluded subjects with psychiatric disorders or chronic diseases capable of impacting on cognition (such as, diabetes mellitus, asthma and sickle cell disease), along with alcoholics and those who had previously used (or smoked) substances other than tobacco, such as cannabis, cocaine, and heroin. Users of electronic cigarettes were also excluded.

The ethical approval for this study was obtained from the Institutional Review Board of IAU under the reference number IRB-PGS-2018-01-033. The research was according to the principles of Helsinki Declaration. All participants received a detailed information form 
accompanied by a verbal explanation of the procedure. Written consent was obtained from all participants prior to commencement of the study. All participants filled out a self-designed questionnaire comprised of questions related to their smoking and health status. The participants were then assessed for their cognitive functions by means of the Cambridge neuropsychological test automated battery (CANTAB).${ }^{15}$ In addition, blood samples were collected and plasma levels of BDNF were measured.

Self-developed questionnaire. The questionnaire was designed to collect information concerning the participants' health status and history of smoking. All participants answered questions about their: i) age; ii) chronic diseases; ii) psychiatric disorders; iv) use of medication; v) smoking; vi) use of alcohol; and vii) use of addictive substances other than tobacco. Smokers were asked additional questions related to their smoking history (such as, age of their initiation into smoking, the number of cigarettes smoked per day and current or past smoking of tobacco products other than cigarettes). Pack-years for smokers (number of cigarettes smoked per day $\times$ years as a smoker/20) was calculated to quantify their lifetime exposure to cigarette smoking. A pilot study was conducted with 18 participants (9 smokers and 9 non-smokers) aged between 24 and 34 , to test the clarity of the tool and estimate the time required to complete the questionnaire. The piloted participants were not involved, and few changes were made in the final questionnaire in response to the pilot study. The finalized questionnaire used in the study is available in Appendix A.

Cognitive assessment. Cambridge neuropsychological test automated battery is a battery of computerized neuropsychological tests by University of Cambridge, England. ${ }^{15}$ The CANTAB tests depend on touch screen technology, which provides rapid and non-invasive cognitive assessment. These tests follow a game-like format, in which a feedback is provided following each trial, thus maintaining the motivation of the participants. $^{15}$

Four tests from CANTAB were used to assess the cognitive functions of the current participants, namely: i) reaction time (RTI); ii) spatial working memory (SWM); iii) rapid visual information processing (RVP); and iv) multitasking test (MTT). Testing took place in a quiet room, with the subjects provided an iPad (Apple Inc, USA) and headphones. All the participants received a detailed explanation on how to undertake each cognitive test and the tests were all carried out in the same sequence for each of the subjects.
Reaction time. This test is used to assess mental and motor response speeds and measures reaction time, impulsivity, movement time and response accuracy. This study measured RTI median 5-choice movement time and RTI median 5-choice reaction time.

Spatial working memory. This consists of an executive function and decision-making test used to assess spatial working memory. The test was used to measure spatial working memory between errors (SWMBE468) and SWMS 6-8 boxes.

Rapid visual information processing. This test is used to evaluate attention, in particular sustained attention. It was used to measure rapid visual-information processing (RVPA), rapid visual-information processing median response latency and rapid visual-information processing probability of false alarm (RVPPFA).

Multitasking test. This is an executive function and decision-making test assessing the ability to manage conflicting information and ignore task-irrelevant information. It was used to measure multitasking test reaction latency (median), multitasking test incongruency cost (median), multitasking test multitasking cost (median), and MTT total incorrect.

Brain-derived neurotrophic factor sandwich enzymelinked immuno-sorbent assay (ELISA). Approximately 3 milliliters of venous blood was collected in a tripotassium ethylene diamine tetra-acetic acid (EDTA. K3) tube and centrifuged for 15 minutes ( $\mathrm{min}$ ) at $1,500 \times \mathrm{g}$ within $45 \mathrm{~min}$ of collection. Following centrifugation, the plasma portion was aliquoted into an Eppendorf tube and stored at $-80^{\circ} \mathrm{C}$. Measurements of plasma BDNF concentrations were carried out according to the manufacturer's instructions in our institutional biochemistry laboratory, using human BDNF sandwich ELISA kits (Aviva systems biology, CA, USA).

Statistical analysis. The data were entered and analyzed using IBM SPSS Statistics for Windows, version 24 (IBM Corp, Armonk, NY, USA). Testing for normality of distribution was performed using the Shapiro-Wilk test. A comparison between the 2 groups (such as, smokers and non-smokers) was undertaken by means of an unpaired student t-test for parametric data and a Mann-Whitney test for non-parametric data. Mean and standard deviations were calculated for parametric variables (RTI, SWM, MTI , and BDNF), with median and range being calculated for non-parametric variables (RVP). A Chi-square $(\chi 2)$ test was used for qualitative data analysis. To determine the association between smoking related variables (such as, age at commencement of smoking; number of cigarettes/day; duration of smoking; and the number 
of pack-years) and cognitive test scores and BDNF concentrations, Pearson's correlation was used for parametric data and spearman's correlation was used for non-parametric data. Mean values and standard deviations were also calculated and a $p$-value of $<0.05$ was taken as statistically significant.

Results. Of the 73 participants, $42.5 \%(n=31)$ were smokers and $57.5 \%(\mathrm{n}=42)$ were non-smokers. The mean age of smokers was $24.7 \pm 4.1$ years, compared to $20.9 \pm 2.7$ years for non-smokers (Table 1 ).

Cognitive function. Significant difference was observed between smokers and non-smokers in 3 cognitive test scores related to RVP test and MTT. Non-smokers performed significantly better than smokers in RVPA $(p=0.001)$, and RVPPFA $(p=0.027)$ and MTTLMD ( $p=0.007)$ (Table 2).

No significant associations were observed between smoking related variables and cognitive test scores (Table 3).

Plasma BDNF levels. No statistically significant difference was found between the 2 groups with regards to plasma BDNF levels (Table 2). A statistically significant and moderately positive correlation was observed between plasma levels of BDNF and the number of cigarettes/day $(\mathrm{r}=0.480, p=0.024)$. However, no significant association was observed between BDNF and other smoking related variables (example, starting age, duration of smoking, and number of packs per year) (Table 3).

Discussion. This study compared the cognitive functioning and plasma BDNF levels of healthy young smokers and non-smokers, to establish the association

Table 1 - Characteristics of study participants (N=73).

\begin{tabular}{lcccc}
\hline \multirow{2}{*}{ Demographics } & \multicolumn{2}{c}{ Smokers $(\mathrm{n}=31)$} & \multicolumn{2}{c}{ Non-smokers $(\mathrm{n}=42)$} \\
& Mean \pm SD & Range & Mean \pm SD & Range \\
\hline Age (years) & $24.7 \pm 4.1$ & $18-33$ & $20.9 \pm 2.7$ & $17-26$ \\
$\begin{array}{l}\text { Age at starting } \\
\text { smoking } \\
\text { (years) }\end{array}$ & $17.4 \pm 3.3$ & $8-24$ & & \\
$\begin{array}{l}\text { Number of } \\
\text { cigarettes/day }\end{array}$ & $14.8 \pm 9.2$ & $1-40$ & \\
$\begin{array}{l}\text { Duration } \\
\text { of smoking }\end{array}$ & $7.3 \pm 5$ & $1-21$ & \\
$\begin{array}{l}\text { (years) } \\
\text { Pack-years }\end{array}$ & $6.44 \pm 8.35$ & $0.15-40$ & \\
\hline
\end{tabular}

between cigarette smoking and cognition and plasma BDNF levels. We found that young healthy smokers demonstrated impaired sustained attention and ability to manage conflicting information while ignoring task-irrelevant information (an executive function). However, no significant difference was identified between the plasma BDNF level of smokers and nonsmokers. In addition, no significant association was observed between smoking-related variables and the cognitive performance of smokers. Finally, we found only a moderately positive association between plasma BDNF levels and number of cigarettes smoked per day.

Smoking and cognition. The finding of impairment of sustained attention in young smokers is consistent

Table 2 - Comparison of cognitive tests' scores and plasma BDNF levels between smokers and non-smokers $(\mathrm{N}=73)$.

\begin{tabular}{|c|c|c|c|}
\hline Variables & Smokers $(\mathrm{n}=31)$ & $\begin{array}{c}\text { Non-Smokers } \\
(\mathrm{n}=42)\end{array}$ & $P$-value \\
\hline \multicolumn{4}{|l|}{ Reaction time } \\
\hline RTIFMDMT & $201.63 \pm 41.64$ & $198.91 \pm 38.54$ & 0.784 \\
\hline RTIFMDRT & $368.09 \pm 37.06$ & $356.30 \pm 34.61$ & 0.186 \\
\hline \multicolumn{4}{|c|}{ Spatial working memory } \\
\hline SWMBE468 & $7.41 \pm 7.48$ & $7.29 \pm 8.34$ & 0.954 \\
\hline SWMS & $7.04 \pm 2.32$ & $5.90 \pm 2.95$ & 0.098 \\
\hline \multicolumn{4}{|c|}{ Rapid visual information processing } \\
\hline RVPA & $0.869(0.087)$ & $0.909(0.050)$ & $0.001^{*}$ \\
\hline RVPMDL & $443(102.3)$ & $426(53.8)$ & 0.308 \\
\hline RVPPFA & $0.0063(0.1102)$ & $0.0058(0.0068)$ & $0.027^{*}$ \\
\hline \multicolumn{4}{|l|}{ Multitasking test } \\
\hline MTTICMD & $65.80 \pm 48.64$ & $46.47 \pm 41.72$ & 0.093 \\
\hline MTTLMD & $620.7 \pm 109.4$ & $555.97 \pm 77.45$ & $0.007^{*}$ \\
\hline MTTMTCMD & $212.38 \pm 179.49$ & $145.0 \pm 90.51$ & 0.091 \\
\hline MTTTIC & $7.32 \pm 8.47$ & $3.73 \pm 3.54$ & 0.053 \\
\hline $\mathrm{BDNF}(\mathrm{pg} / \mathrm{ml})$ & $338.44 \pm 266.4$ & $281.91 \pm 141.1$ & 0.245 \\
\hline \multicolumn{4}{|c|}{$\begin{array}{l}\text { Data presented as mean } \pm \text { SD or median }(\mathrm{IQR}) \text { as appropriate. } \\
\text { RTIFMDMT: reaction time median five-choice movement time, } \\
\text { RTIFMDRT: reaction time median five-choice reaction ime, SWM: } \\
\text { spatial working memory, SWMBE468: spatial working memory between } \\
\text { errors; SWMS: spatial working memory strategy, RVP: rapid visual } \\
\text { information processing, RVPA: rapid visual information processing A', } \\
\text { RVPMDL: rapid visual information processing median response latency, } \\
\text { RVPPFA: rapid visual information processing probability of false Alarm, } \\
\text { MTTICMD: multitasking test incongruency cost (median), } \\
\text { MTTLMD: multitasking test reaction latency (median), } \\
\text { MTTMTCMD: multitasking test multitasking cost (median); } \\
\text { MTTTIC: multitasking test total incorrect, BDNF: brain derived } \\
\text { neurotrophic factor, *Statistically significant difference }(p<0.05) \text {. }\end{array}$} \\
\hline
\end{tabular}


Table 3 - Correlations between smoking related variables and cognitive test scores and plasma BDNF level (N=31).

\begin{tabular}{|c|c|c|c|c|c|c|c|c|}
\hline \multirow{2}{*}{ Variables } & \multicolumn{2}{|c|}{ Age at starting smoking } & \multicolumn{2}{|c|}{ Cigarettes/day } & \multicolumn{2}{|c|}{ Duration of smoking (years) ${ }^{\dagger}$} & \multicolumn{2}{|c|}{ Pack-years ${ }^{\ddagger}$} \\
\hline & $\mathbf{r}$ & $P$-values & $\mathbf{r}$ & $P$-values & $\mathbf{r}$ & $P$-values & $\mathbf{r}$ & $P$-values \\
\hline \multicolumn{9}{|l|}{ Reaction time } \\
\hline RTIFMDMT & -0.018 & 0.929 & -0.072 & 0.726 & -0.158 & 0.431 & -0.173 & 0.399 \\
\hline RTIFMDRT & 0.109 & 0.589 & -0.103 & 0.615 & -0.181 & 0.367 & -0.144 & 0.482 \\
\hline \multicolumn{9}{|c|}{ Spatial working memory } \\
\hline SWMBE468 & -0.182 & 0.363 & -0.046 & 0.824 & 0.169 & 0.400 & 0.036 & 0.862 \\
\hline SWMS & 0.322 & 0.102 & -0.045 & 0.828 & -0.168 & 0.401 & -0.125 & 0.543 \\
\hline \multicolumn{9}{|c|}{ Rapid visual information processing } \\
\hline RVPA & 0.289 & 0.161 & -0.070 & 0.745 & -0.083 & 0.692 & -0.112 & 0.603 \\
\hline RVPMDL & -0.043 & 0.839 & 0.306 & 0.145 & -0.037 & 0.859 & 0.240 & 0.259 \\
\hline RVPPFA & -0.092 & 0.662 & 0.025 & 0.909 & 0.031 & 0.882 & 0.078 & 0.717 \\
\hline \multicolumn{9}{|l|}{ Multitasking test } \\
\hline MTTICMD & -0.163 & 0.436 & 0.218 & 0.307 & 0.147 & 0.482 & 0.236 & 0.268 \\
\hline MTTLMD & 0.237 & 0.254 & 0.252 & 0.235 & 0.015 & 0.944 & 0.054 & 0.802 \\
\hline MTTMTCMD & 0.283 & 0.171 & -0.003 & 0.987 & -0.027 & 0.897 & 0.007 & 0.975 \\
\hline MTTTIC & 0.147 & 0.482 & 0.270 & 0.201 & 0.035 & 0.869 & 0.064 & 0.766 \\
\hline BDNF (pg/ml) & -0.129 & 0.558 & 0.480 & $0.024^{*}$ & 0.294 & 0.173 & 0.375 & 0.085 \\
\hline $\begin{array}{r}\text { Data presented as } \\
\text { RTIFMDRT: reac } \\
\text { SWMS: } \\
\text { RVPMDL: rapid } \\
\text { MTTICMD: multit } \\
\text { multitasking cost } \\
(p\end{array}$ & $\begin{array}{l}\text { SD or me } \\
\text { ne median } \\
\text { orking me } \\
\text { informatic } \\
\text { test incon } \\
\text {, MTTT } \\
\text { 'Duratio } \\
\quad{ }^{\ddagger} \mathrm{Pac}\end{array}$ & $\begin{array}{l}\text { (IQR) as ap } \\
\text { oice reactio } \\
\text { y strategy, } \mathrm{R} \\
\text { ocessing me } \\
\text { cy cost (me } \\
\text { hultitasking } \\
\text { moking was } \\
\text { ars were calc }\end{array}$ & $\begin{array}{l}\text { priate. RT } \\
\text { me, SWN } \\
\text { rapid vis } \\
\text { response } \\
\text {, MTTL } \\
\text { total inco } \\
\text { culated by } \\
\text { ed as [the }\end{array}$ & $\begin{array}{l}\text { action tim } \\
\text { atial worki } \\
\text { formation } \\
\text { ncy, RVPP } \\
\text { : multitask } \\
\text {, BDNF: } \\
\text { tracting ag } \\
\text { nber of cig }\end{array}$ & $\begin{array}{l}\text { IFMDMT, } \\
\text { emory, SW } \\
\text { cessing, RV } \\
\text { apid visual } \\
\text { est reaction } \\
\text { derived net } \\
\text { starting smo } \\
\text { es/day } \times \text { yea }\end{array}$ & $\begin{array}{l}\text { time media } \\
\text { : spatial wor } \\
\text { t visual infor } \\
\text { tion processi } \\
\text { (median), M } \\
\text { ic factor, }{ }^{*} \text { St } \\
\text { m participan } \\
\text { noker } / 20 \text { ]. }\end{array}$ & $\begin{array}{l}\text { choice mo } \\
\text { memory b } \\
\text { n process } \\
\text { bability c } \\
\text { TCMD: } \\
\text { ally signif } \\
\text { ent age. }\end{array}$ & $\begin{array}{l}\text { ent time, } \\
\text { en errors, } \\
\text { e alarm, } \\
\text { itasking tes } \\
\text { difference }\end{array}$ \\
\hline
\end{tabular}

with the results of previous study conducted by Vajravelu et al. ${ }^{16}$ In their study, they reported impaired attention in moderate and heavy smokers only. Mild smokers demonstrated improved sustained attention and alertness over non-smokers. ${ }^{16}$

In executive functioning, we found that, in comparison to non-smokers, smokers demonstrated an impaired ability to manage conflicting information and ignore task-irrelevant information. Our results agree with the previous studies that have reported impairment in executive functions in middle aged and elderly smokers. ${ }^{17,18}$

The insignificant difference in spatial working memory and reaction time tests between young smokers and non-smokers is inconsistent with a previous study conducted on young individuals. ${ }^{16}$ This discrepancy in results could be due to our small sample size or presence of a number of confounding factors we were unable to control, example, baseline cognitive ability, age, education, and the use of caffeinated beverages prior to the cognitive assessment.

Lack of association between smoking and cognitive performance, as found in our study, is inconsistent with studies conducted before. ${ }^{19-21}$ Studies by Depp et $\mathrm{al}^{19}$ and Hickling et $\mathrm{al}^{20}$ were cross-sectional; whereas Vermeulen et $\mathrm{al}^{21}$ conducted a large prospective study to explore the association between smoking behavior and cognition at baseline, at 3-6-year follow-ups. All these studies reported an inverse relationship between smoking and cognition.

Smoking and plasma BDNF levels. We did not find any significant difference in plasma BDNF levels of smokers compared to non-smokers; a finding contrary to Neves et $\mathrm{al}^{22}$ who reported significantly higher BDNF in heavy smokers compared to non-smokers. The underlying cause of discrepancy could be due to 
the possibility that our study participants were lightsmokers and not the heavy-smokers. Nevers et $\mathrm{al}^{22}$ failed to observe any significant difference in BDNF levels of mild smokers compared to non-smokers. We found a moderately positive association between plasma BDNF levels and the number of cigarettes/days. This is consistent with Colle et $\mathrm{al}^{23}$ who reported a positive correlation of plasma BDNF with number of cigarettes/ day and number of pack/years. Zhang et $\mathrm{al}^{24}$ also reported smoking severity to be positively associated with BDNF levels. This positive association between plasma BDNF levels and the number of cigarettes/days could also be indicative of a compensatory neuroprotective mechanism, or an underlying development and maintenance of nicotine dependence.

Future studies are required to examine the relationship between smoking and BDNF levels in the brain and to explore the mechanisms underlying this relationship and to enhance understanding of the interrelationship between smoking, cognition, addiction and the BDNF system.

Study limitations. We used a relatively small sample size. The lack of statistically significant correlations may be due to small sample size leading to low power; the correlation between RVPA and age at start $(\mathrm{r}=0.289)$ : the power is as low as 35\%.

The $p$-values are also affected by the sample size (smaller the sample size, larger is the $p$-values). Our study was limited to male subjects only. The participants were recruited by non-probability sampling technique, and they may not have been representative of the entire population. This was an observational study and so did not allow for examination of genetic associations or neurobiological mechanisms underlying the influence of smoking on cognition. Self-reported medical, psychiatric, smoking, medication, and addiction histories were recorded that were not based on physical or laboratory examinations. We did not assess any other confounders that may influence cognition or BDNF levels, example nutrition, consumption of caffeinated beverages, and passive smoking, and so on.

In conclusion, young smokers have significantly impaired sustained attention and less ability to manage conflicting information as compared to age-matched non-smokers. Plasma BDNF level is positively related to the number of cigarettes smoked per day. No association was found between plasma BDNF levels and the starting age of smoking, duration of smoking and the number of pack-years.
Acknowledgment. The authors gratefully acknowledge Academic Proofreader LTD. Company (www.academicproofreader. com) for English language editing.

\section{References}

1. World Health Organization. WHO report on the global tobacco epidemic, 2015 Raising taxes on tobacco, Geneva, Switzerland: World Health Organization. [Updated 2018. Accessed 2018 January 15]. Available from URL: https://www. who.int/tobacco/global_report/2015/en/

2. Moradi-Lakeh M, El Bcheraoui C, Tuffaha M, Daoud F, Al Saeedi M, Basulaiman M, et al. Tobacco consumption in the Kingdom of Saudi Arabia, 2013: findings from a national survey. BMC Public Health 2015; 15: 611.

3. World Health Organization. World No Tobacco Day 2018: Tobacco breaks hearts. World Health Organization. [Updated 2018. Accessed 2018 January 15]. Available from: http:// www.emro.who.int/media/news/world-no-tobacco-day-2018tobacco-breaks-hearts.html

4. West R. Tobacco smoking: Health impact, prevalence, correlates and interventions. Psychol Health 2017; 32: 1018-1036.

5. Chan YL, Saad S, Pollock C, Oliver B, Al-Odat I, Zaky AA, et al. Impact of maternal cigarette smoke exposure on brain inflammation and oxidative stress in male mice offspring. Sci Rep 2016; 6: 1-12.

6. Song Y, Kim J Goo, Cho HJ, Kim JK, Suh DC. Evaluation of cerebral blood flow change after cigarette smoking using quantitative MRA. PLoS One 2017; 12: e0184551.

7. Chen S, Wu P, Zhou L, Shen Y, Li Y, Song H. Relationship between increase of serum homocysteine caused by smoking and oxidative damage in elderly patients with cardiovascular disease. Int J Clin Exp Med 2015; 8: 4446-4454.

8. Karama S, Ducharme S, Corley J, Chouinard-Decorte F, Starr JM, Wardlaw JM, et al. Cigarette smoking and thinning of the brain's cortex. Mol Psychiatry 2015; 20: 778-785.

9. Valentine G, Sofuoglu M. Cognitive effects of nicotine: recent progress. Curr Neuropharmacol 2018; 16: 403-414.

10. Khorasanchi Z, Bahrami A, Avan A, Jaberi N, Rezaey M, Bahrami-Taghanaki $\mathrm{H}$, et al. Passive smoking is associated with cognitive and emotional impairment in adolescent girls. J Gen Psychol 2019; 146: 68-78.

11. Miranda M, Morici JF, Zanoni MB, Bekinschtein P. BrainDerived neurotrophic factor: a key molecule for memory in the healthy and the pathological brain. Front Cell Neurosci 2019; 13: 363 .

12. Buhusi M, Etheredge C, Granholm AC, Buhusi CV. Increased hippocampal proBDNF contributes to memory impairments in aged mice. Front Aging Neurosci 2017; 9: 284.

13. Campos MW, Serebrisky D, Castaldelli-Maia JM. Smoking and Cognition. Curr Drug Abuse Rev 2016; 9: 76-79.

14. Dean AG, Sullivan KM, Soe MM. OpenEpi: open source epidemiologic statistics for public health, version 3.01 . (Updated 2014. Accessed 2018 January 15). Available from URL: https://www.openepi.com/Menu/OE_Menu.htm

15. Smith PJ, Need AC, Cirulli ET, Chiba-Falek O, Attix DK. A comparison of the Cambridge automated neuropsychological test battery (CANTAB) with 'traditional' neuropsychological testing instruments. J Clin Exp Neuropsychol 2013; 35: 319-328. 
16. Vajravelu HR, Gnanadurai TK, Krishnan P, Ayyavoo S. Impact of quantified smoking status on cognition in young adults. $J$ Clin Diagnostic Res 2015; 9: CC01-CC03.

17. McClernon FJ, Froeliger B, Rose JE, Kozink RV, Addicott MA, Sweitzer MM, et al. The effects of nicotine and non-nicotine smoking factors on working memory and associated brain function. Addict Biol 2016; 21: 954-961.

18. Heffernan TM, Carling A, O’Neill TS, Hamilton C. Smoking impedes executive function and related prospective memory. Ir J Psychol Med 2014; 31: 159-165.

19. Depp CA, Bowie CR, Mausbach BT, Wolyniec P, Thornquist $\mathrm{MH}$, Luke JR, et al. Current smoking is associated with worse cognitive and adaptive functioning in serious mental illness. Acta Psychiatr Scand 2015; 131: 333-341.

20. Hickling LM, Perez-Iglesias R, Ortiz-García de la Foz V, Balanzá-Martínez V, McGuire P, Crespo-Facorro B, et al. Tobacco smoking and its association with cognition in first episode psychosis patients. Schizophr Res 2018; 192: 269-273.
21. Vermeulen JM, Schirmbeck F, Blankers M, van Tricht M, Bruggeman R, van den Brink W, et al. Association between smoking behavior and cognitive functioning in patients with psychosis, siblings, and healthy control subjects: results from a prospective 6-year follow-up study. Am J Psychiatry 2018; 175: 1121-1128.

22. Neves CDC, Lacerda ACR, Lima LP, Lage VKS, Balthazar CH, Leite HR, et al. Different levels of brain-derived neurotrophic factor and cortisol in healthy heavy smokers. Braz J Med Biol Res 2017; 50: e6424.

23. Colle R, Trabado S, Rotenberg S, Brailly-Tabard S, Benyamina A, Aubin HJ, et al. Tobacco use is associated with increased plasma BDNF levels in depressed patients. Psychiatry Res 2016; 246: 370-372.

24. Zhang XY, Tan Y, Chen D, Tan SP, Yang FD, Zunta-Soares $\mathrm{GB}$, et al. Effects of cigarette smoking and alcohol use on neurocognition and BDNF levels in a Chinese population. Psychopharmacology (Berl) 2016; 233: 435-445.

Appendix A - Self-made questionnaire (for current smokers and non-smokers).

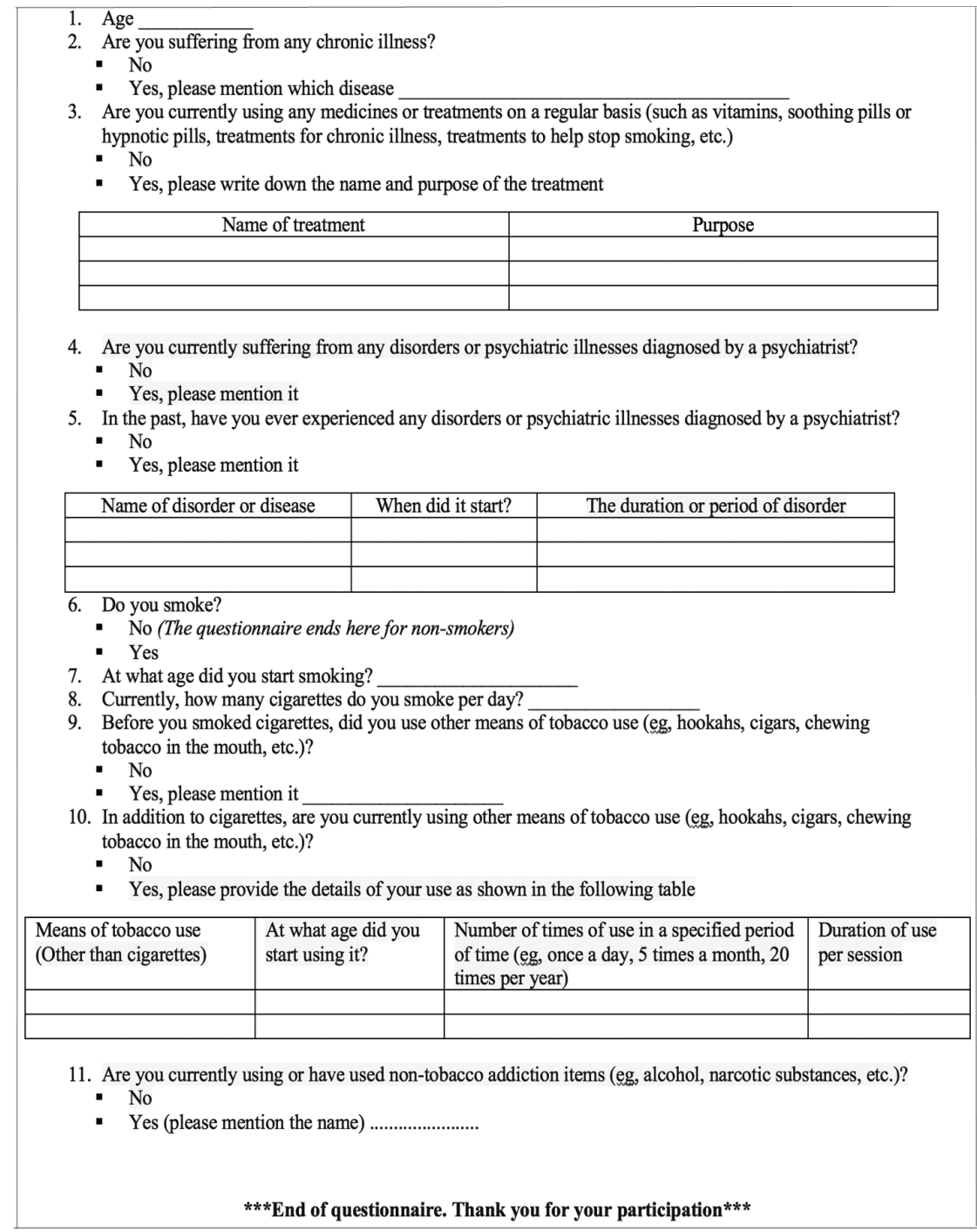

